\title{
Role of Advanced Glycation End Products on Aortic Calcification in Patients with Type 2 Diabetes Mellitus
}

\author{
Pilar Sanchis $1,2,3, * \mathbb{0}$, Rosmeri Rivera ${ }^{1}$, Regina Fortuny ${ }^{1,4}$, Carlos Río ${ }^{5}$, Miguel Mas-Gelabert ${ }^{6}$, \\ Marta Gonzalez-Freire ${ }^{1}$, Felix Grases ${ }^{2,3}$ (1) and Luis Masmiquel ${ }^{1, *}$ \\ 1 Vascular and Metabolic Diseases Research Group, Endocrinology Department, Son Llàtzer University \\ Hospital, Health Research Institute of the Balearic Islands [IUNICS-IdISBa], 07198 Palma of Mallorca, Spain; \\ rosmeri_ri@hotmail.com (R.R.); rfortuny@hsll.es (R.F.); marta.gonzalezfreire@ssib.es (M.G.-F.) \\ 2 Laboratory of Renal Lithiasis Research, Deptartment of Chemistry, University of Balearic Islands, \\ Health Research Institute of the Balearic Islands [IUNICS-IdISBa], 07122 Palma of Mallorca, Spain; \\ fgrases@uib.es \\ 3 The Spanish Biomedical Research Centre in Physiopathology of Obesity and Nutrition (CIBERObn), \\ Instituto de Salud Carlos III, 28046 Madrid, Spain \\ 4 Laboratory Department, Son Llàtzer University Hospital, 07198 Palma of Mallorca, Spain \\ 5 Proteomics department, Health Research Institute of the Balearic Islands (IdISBa), \\ 07120 Palma of Mallorca, Spain; carlos.rio@ssib.es \\ 6 Radiology Department, Son Llàtzer University Hospital, 07198 Palma of Mallorca, Spain; mmas1@hsll.es \\ * Correspondence: pilar.sanchis@uib.es (P.S.); lmasmiquel@hsll.es (L.M.)
}

Received: 19 May 2020; Accepted: 2 June 2020; Published: 5 June 2020

\begin{abstract}
The aim of this study was to evaluate the relationship between serum levels of advanced glycation end products (AGEs) and abdominal aortic calcification (AAC) in patients with type 2 diabetes mellitus (DM2). This was a prospective cross-sectional study. One-hundred and four consecutive patients with DM2 were given lateral lumbar X-rays in order to quantify abdominal aortic calcification (AAC). Circulating levels of AGEs and classical cardiovascular risk factors were determined. Clinical history was also registered. Patients with higher AGEs values had higher grades of aortic calcification and higher numbers of diabetic-related complications. Multivariate logistic regression analysis showed that being older, male and having high levels of AGEs and triglycerides were the independent risk factors associated to moderate-severe AAC when compared to no-mild AAC. Our results suggest that AGEs plays a role in the pathogenesis of aortic calcifications. In addition, the measurement of AGEs levels may be useful for assessing the severity of AAC in the setting of diabetic complications.
\end{abstract}

Keywords: AGEs; aortic calcification; type 2 diabetes mellitus; diabetes-related complications

\section{Introduction}

Cardiovascular $(\mathrm{CV})$ disease is the leading cause of death in patients with type 2 diabetes mellitus (DM2) in Western countries and represents a major burden on morbidity, quality of life and health resources. Thus, the identification of patients with DM2 at a high risk of a CV event is important to develop early interventions and prevention strategies. In clinical practice, patients with worse lipid profiles, higher blood pressure, obesity, hyperglycemia, cardiac hypertrophy or dysfunction, nephropathy, neuropathy and/or retinopathy tend to have a higher prevalence of CVD [1]. In recent years, however, several studies have suggested that abdominal aortic calcification (AAC) increases the risk of major CV events in patients with DM2 [2-4]. AAC has been related to severe coronary artery calcification and cardiovascular outcomes specially in patients with DM2 and chronic kidney disease $[5,6]$. Also, it has been reported that AAC predicts major CV events after an acute coronary 
syndrome and predicts health care costs in older men independent of CV disease status as well $[7,8]$. Hence, detection and scoring AAC could improve the clinical identification of patients with DM2 at risk of developing major $\mathrm{CV}$ events.

AAC is described as the hardening of the medial layer of the artery through deposition of hydroxyapatite crystals into the extracellular matrix [9-11]. Calcification occurs, amongst other factors, as a consequence of aging [12], but it can be accelerated by other diseases like renal insufficiency and diabetes $[13,14]$. Although several factors could accelerate or arrest the natural process of vascular calcification, the pathogenesis of vascular calcification in diabetes is not completely understood. Among the list of inducers, we can find hyperphosphatemia, vitamin D, lipids or inflammatory cytokines, whereas fetuin-A, phytate, pyrophosphate, vitamin $\mathrm{K}$, osteopontin or matrix Gla protein have been described to be inhibitors of vascular calcification [15]. Consequently, further understanding of the mechanisms by which diabetes might favor this process needs to be elucidated. Indeed, high glucose appears to have an important role in the initiation and progression of this complication.

Ideally, AAC can be quantified with computed tomography (CT), lumbar X-ray and dual-energy X-ray absorptiometry (DXA) scans [16]; however, these imaging studies are not routinely performed in the clinic. Therefore, circulating biomarkers could be useful to identify those patients more prone to AAC and to develop new treatments. In this regard, low-density lipoproteins (LDL), high-density lipoproteins (HDL), glycated hemoglobin ( $\mathrm{HbA1c})$, osteocalcin, phosphate and advanced glycation end products (AGEs) have been identified as potential markers of AAC but literature is ambiguous and further research is needed [2].

AGEs are the irreversible products of the reaction between amino groups of proteins and sugar resulting from long-term hyperglycemia [17]. AGEs are formed at an accelerate rate under non-controlled diabetic state and play an important role in the development and progression of vascular complications in diabetes [18]. AGEs can affect cell function via intracellular glycation of proteins [19], provoke arterial stiffness by cross-linking of extracellular matrix proteins in arteries [20], and induce receptor-mediated cell activation by binding to the receptor of AGEs (RAGE) [21]. AGEs are believed to be involved in the onset and progression of atherosclerosis through multiple mechanisms and several studies indicate that AGEs and their receptors (RAGEs) play an important role in vascular calcification [22]. In individuals with chronic kidney disease (CKD), AGEs have been associated with arterial calcification [23,24]. Recently, several studies using different models of vascular smooth muscle cells (VSMC) demonstrated the implication of AGEs in calcification [25-28]. Indeed, it was shown that exposure of VSMC to AGEs induced cell calcification [29]. However large studies investigating plasma AGEs levels and their relation with AAC are scarce.

Therefore, the main objectives of the present study are: (1) to examine the association between serum levels of AGEs with AAC, quantified on lumbar X-ray; and (2) to determine the most important $\mathrm{CV}$ risk factors and diabetic complications associated to moderate-severe AAC in T2DM.

\section{Experimental Section}

\subsection{Subjects and Methods}

One-hundred and four patients with DM2 were recruited consecutively at the Outpatient Diabetic Clinic of Son Llàtzer University Hospital (Palma de Mallorca, Spain). The demographic characteristics of the patients according to the AGEs levels are shown in Table 1. Briefly, patients were included if they were older than 40 years, had DM2 duration longer than 1 year, and had had a lumbar X-ray performed in the past 6 months. Exclusion criteria were: life expectancy shorter than 1 year, immunodeficiency, human immunodeficiency virus (HIV) infection, drug abuse or alcohol intake higher than $50 \mathrm{~g} /$ day, body mass index (BMI) higher than $40 \mathrm{Kg} / \mathrm{m}^{2}$, participation in a clinical trial in the past year, inability to attend programmed visits, illiteracy, chronic or acute infection in the past 3 months, neoplasia in the past 5 years, vasculitis, resistant hypertension, moderate or severe hepatic insufficiency or glomerular filtration rate (GFR) lower than $30 \mathrm{~mL} / \mathrm{min} / 1.73 \mathrm{~m}^{2}$. 
Table 1. Clinical characteristics of patients among circulating advanced glycation end products (AGEs) groups.

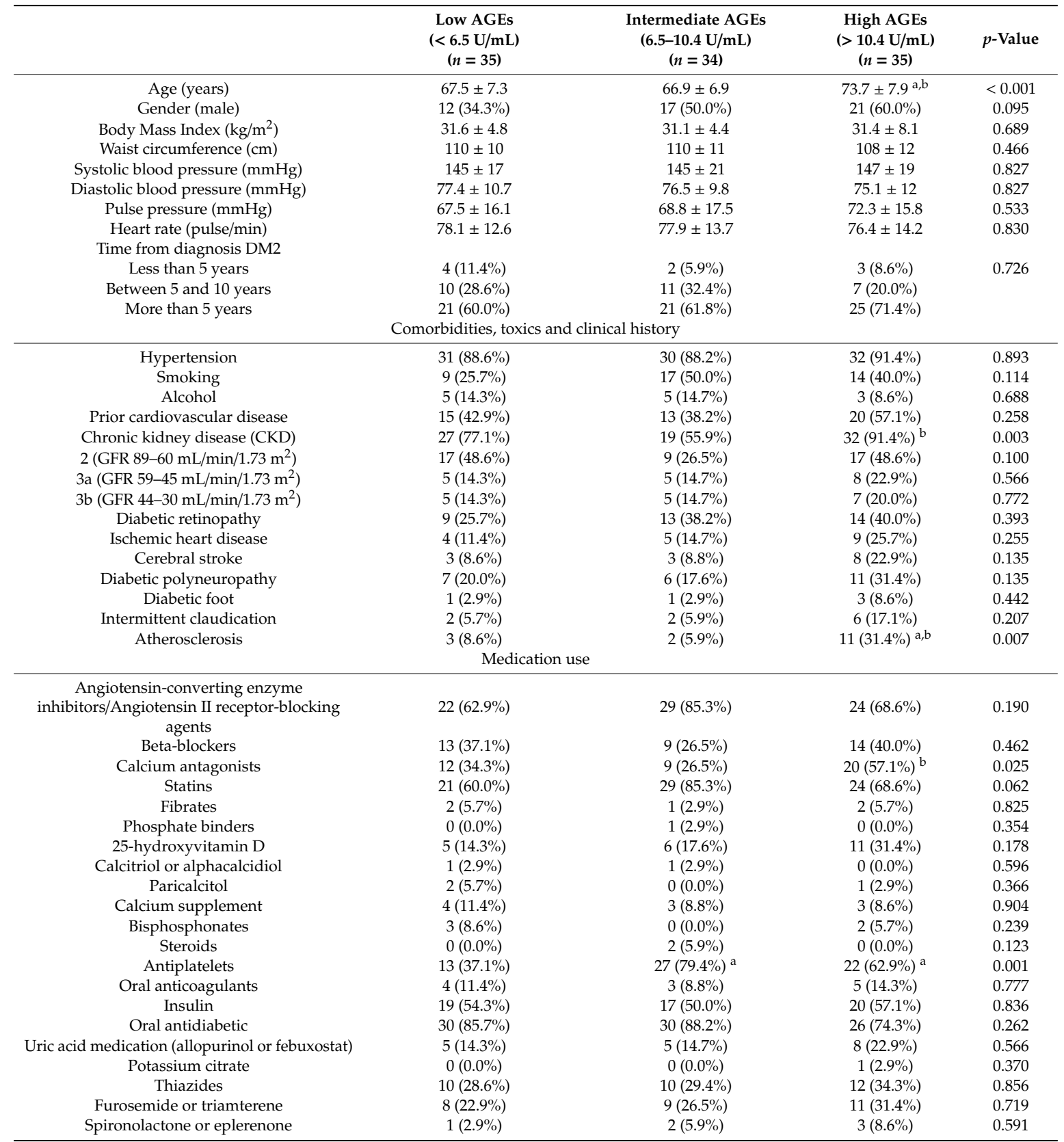

Each value is given as mean \pm standard deviation or frequency (percentage). The significance of differences between groups were determined using one-way analysis of variance (ANOVA) and the independent-samples $t$-test; or Kruskal-Wallis test and Mann-Whitney U test for quantitative data. Chi-square test and Fisher's exact test were used for qualitative data. a: $p<0.05$ vs. corresponding value of Low AGEs group; $b: p<0.05$ vs. corresponding value of Intermediate AGEs group. Abbreviations. DM2: type 2 diabetes mellitus; GFR: glomerular filtration rate calculated by MDRD-4 IDMS equation.

The study protocol was approved by the Ethics Committee of the Balearic Islands (approval number: CEIC-IB 2523/15PI). Written informed consent was obtained from all study participants.

\subsection{Variables Outcomes}

The main outcome measures were serum level of AGEs and AAC scores. Clinical histories were obtained from the electronic medical records. Furthermore, data from anamnesis, laboratory analysis, and physical examinations were prospectively collected during the study. Physical and 
anthropometric measurements were determined by qualified personnel. Blood samples were collected in the morning (after $12 \mathrm{~h}$ of fasting). The samples were left to stand for $30 \mathrm{~min}$ at room temperature, and the serum was then separated by centrifugation. Biochemical analyses were performed in an automated analyzer (Cell-Dyn Sapphire and Architect ci16200, Abbott, IL, USA). Insulin was analyzed by chemiluminescent-immunometric assay (Advia Centaur, Siemens, NY, USA). Highly sensitive C-reactive protein (hs-CRP) and lipoprotein (a) (Lp [a]), were analyzed by nephelometry (Immage 8000, Beckman Coulter Inc, CA, USA). All samples were run in duplicate, and the coefficients of intraand inter-assay variation were below $10 \%$.

Blood pressure was measured 3 times consecutively after $5 \mathrm{~min}$ of rest while the subject was sitting quietly. The average of the second and third measurements was recorded. Patients using anti-hypertensive drugs and those with systolic blood pressure of $140 \mathrm{mmHg}$ or more and/or diastolic blood pressure of $90 \mathrm{mmHg}$ or more were categorized as having hypertension [30]. Atherosclerosis was diagnosed by having clinically significant carotid and/or femoral plaque burden documented with arterial ultrasonography. Chronic kidney disease (CKD) was diagnosed based on the estimated GFR (stage 2 CKD: $89-60 \mathrm{~mL} / \mathrm{min} / 1.73 \mathrm{~m}^{2}$; stage 3a CKD: $59-45 \mathrm{~mL} / \mathrm{min} / 1.73 \mathrm{~m}^{2}$; stage 3b: $44-30 \mathrm{~mL} / \mathrm{min} / 1.73 \mathrm{~m}^{2}$ ), calculated as previously described [31].

\subsection{Serum Advanced Glycation End Products (AGEs) Determination}

AGEs in serum samples were measured using the OxiSelect ${ }^{\mathrm{TM}}$ AGE Competitive enzyme-linked immunosorbent assay (ELISA) Kit (Cell Biolabs Inc, CA, USA), which provides rapid detection and quantification of AGE protein adducts. Quantitation was determined by comparing absorbance with that of a known AGE-bovine serum albumin (BSA) standard curve. First, an AGE conjugate was coated on an ELISA plate. The unknown AGE samples or AGE-BSA standards were added to the AGE conjugate pre-absorbed ELISA plate. After a brief incubation, an anti-AGE polyclonal antibody was added, followed by horseradish peroxidase-conjugated secondary antibody. The content of AGE protein adducts in unknown samples was determined by comparison with a pre-determined AGE-BSA standard. The intra-assay coefficient of variation was $6.9 \%$ (three replicates for each sample on the same day). The inter-assay coefficient of variation was 9.2\% (3 days at the same time).

\subsection{Lateral Lumbar Radiography of Abdominal Aorta}

Lateral lumbar X-rays were performed while patients were standing, using standard radiographic equipment. A minimum of $8 \mathrm{~cm}$ of tissue anterior to the lumbar spine, including the abdominal aorta, was visible. The focus-film distance was $100 \mathrm{~cm}$, the tube potential was $94 \mathrm{kV}$, the tube-current-time product was 33-200 mAs, and the estimated radiation dose was approximately $15 \mathrm{mGy}$.

ACC was assessed using a previously validated 24-point scale [32,33]. For this 24-point scale, calcified deposits along the anterior and posterior longitudinal walls of the abdominal aorta, adjacent to each lumbar vertebra (L1 to L4), were assessed using the midpoint of the intervertebral space above and below the vertebrae as the boundaries. Calcifications were graded as 0 (no aortic deposits), 1 (small scattered deposits less than one-third the length of the vertebral length), 2 (intermediate quantity of deposits, about one-third or more, but less than two-thirds of the vertebral length), or 3 (extensive deposits of two-thirds or more of the corresponding vertebral length). The scores were determined separately for the anterior and posterior walls; the range was 0 to 6 for each vertebral level, and 0 to 24 for the total.

All subjects were assessed independently by two graders who were blinded to patient data. To validate the $X$-ray assessment, double readings were performed in all patients demonstrating an excellent inter-observer agreement (intra-class coefficient of correlation $\mathrm{R}=0.948, p<0.0001$ ).

\subsection{Statistical Analysis}

Data are presented as means and standard deviations, medians and interquartile ranges, or numbers and percentages. Patients were divided in three groups according to tertiles of serum 
AGEs levels: low $(\mathrm{L}<6.5 \mathrm{U} / \mathrm{mL})$, intermediate $(\mathrm{I}: 6.5-10.4 \mathrm{U} / \mathrm{mL})$ and high $(\mathrm{H}>10.4 \mathrm{U} / \mathrm{mL})$. Intergroup comparisons of serum levels of AGEs employed one-way analysis of variance (ANOVA) and the independent-samples $t$-test (as post-hoc test); or Kruskal-Wallis test and Mann-Whitney U test (as a post-hoc test) for continuous variables. A chi-square test and Fisher's exact test were used for categorical variables. For the second objective, patients were divided in two groups according the median of AAC scores: no-mild (AAC $<6$ ) and moderate-severe (AAC $\geq 6$ ). Intergroup comparisons of aortic abdominal calcification (AAC) scores employed the independent-samples $t$-test or the Mann-Whitney U test for continuous variables, and the chi-square test or Fisher's exact test for categorical variables. Receiver operating characteristic (ROC) curves of quantitative risk factors associated to moderate-severe AAC were performed. The optimal cutoff values were determined by the maximum Youden index $(\mathrm{J})$, defined as sensitivity + specificity -1 . Binary logistic regression models were used to identify risk factors associated to moderate-severe AAC (AAC $\geq 6$ ), with an AAC less than 6 (no-mild AAC) as the reference (odds ratio [OR] =1). Analysis was performed using the stepwise backward method. A two-tailed $p$-value less than 0.05 was considered statistically significant. Statistical analyses were performed using SPSS 23.0 (SPSS Inc., Chicago, IL, USA).

\section{Results}

\subsection{Baseline Patient Characteristics}

Demographic and clinical characteristics of the three groups are shown in Table 1. Patients with higher AGEs levels were older (L: $67.5 \pm 7.3$; I: $66.9 \pm 10.4$; H: $73.7 \pm 7.9$ years; $p<0.001$ ) and the percentage of males was also higher (L: $34.3 \%$; I: $50.0 \%$ and H: $60.0 \% ; p=0.095$ ), although it did not reach statistical significance. Patients with higher AGEs levels presented more diabetic complications, such as diabetic nephropathy, atherosclerosis and chronic kidney disease $(p<0.05)$. Furthermore, the number of diabetic complications increased with AGEs levels (Figure 1). The percentage of patients with three or more DM complications was $6 \%, 21 \%$ and $34 \%$ for low, intermediate and high AGEs groups, respectively $(p=0.009)$ while the percentage of patients with no DM2 complications were $57 \%$, $38 \%$ and $23 \%$, respectively. Regarding medication, a higher percentage of patients with high AGEs levels were taking calcium antagonists and antiplatelets when compared to patients with low AGEs levels ( $p=0.025$ and $p=0.001$, respectively).

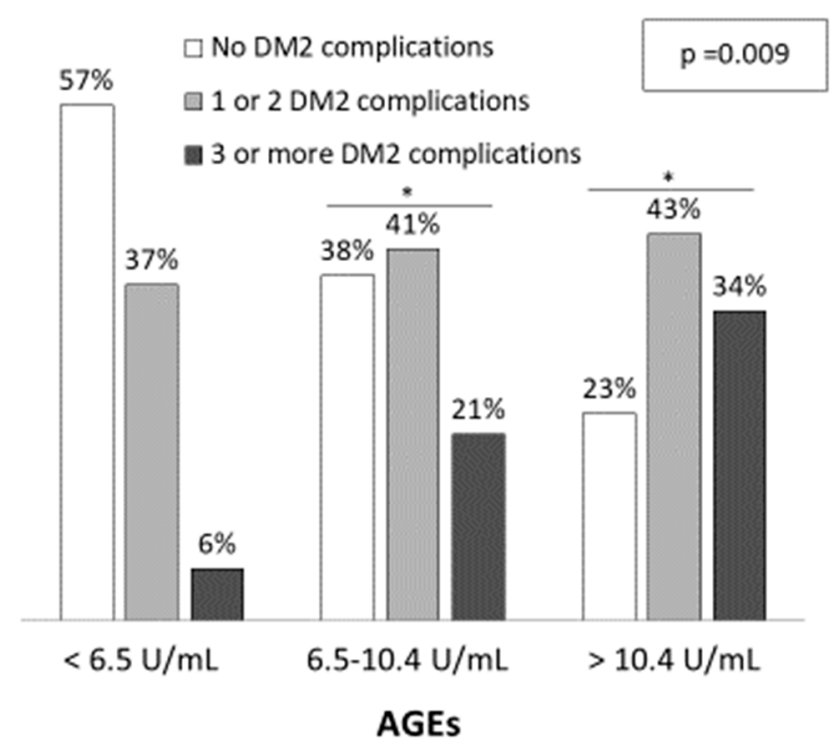

Figure 1. Diabetic related-complications among serum AGEs levels. The significance of differences between groups were determined using chi-square test $(p=0.009) .{ }^{*} p<0.05$ vs. Low AGEs group $(<6.5 \mathrm{U} / \mathrm{mL})$. 


\subsection{Laboratory Analysis Parameters}

Table 2 shows the clinical and biochemical characteristics for AGEs groups. As can be seen, GFR was lower for high AGEs group compared to low and intermediate AGEs groups [L:77.8(55.7-88.9); I:83.1(50.1-98.2); H: 65.4(46.0-79.1) $\mathrm{mL} / \mathrm{min} / 1.73 \mathrm{~m}^{2} ; p=0.040$ ] whereas blood levels of hemoglobin, urea, creatinine, urate, albumin: creatinine ratio and glycated hemoglobin significantly increase with AGEs levels $(p<0.05)$.

Table 2. Laboratory parameters among AGEs groups.

\begin{tabular}{|c|c|c|c|c|}
\hline & $\begin{array}{c}\text { Low AGEs } \\
(<6.5 \mathrm{U} / \mathrm{mL}) \\
(n=35)\end{array}$ & $\begin{array}{c}\text { Intermediate AGEs } \\
(6.5-10.4 \mathrm{U} / \mathrm{mL}) \\
(n=35)\end{array}$ & $\begin{array}{l}\text { High AGEs } \\
(>10.4 \mathrm{U} / \mathrm{mL}) \\
(n=35)\end{array}$ & $p$-Value \\
\hline HbA1c (\%) & $7.2(6.0-8.1)$ & $7.2(6.8-7.8)$ & $8.0(7.0-8.5)^{\mathrm{a}, \mathrm{b}}$ & 0.026 \\
\hline Leukocytes (×109/L) & $7.9(6.7-9.6)$ & $7.5(6.3-9.2)$ & $7.5(6.3-9.5)$ & 0.675 \\
\hline Hemoglobin $(\mathrm{g} / \mathrm{dL})$ & $13.4(12.5-14.5)$ & $13.2(12.6-14.8)$ & $13.0(11.4-13.8)^{a, b}$ & 0.039 \\
\hline Glucose (mg/dL) & $152.0(125.0-194.0)$ & $148.5(125.5-191.8)$ & $138.0(110.0-230.0)$ & 0.847 \\
\hline Urea $(\mathrm{mg} / \mathrm{dL})$ & $38.0(30.0-52.0)$ & $41.0(33.8-48.3)$ & $49.0(37.0-66.0)^{\mathrm{a}}$ & 0.026 \\
\hline Creatinine (mg/dL) & $0.8(0.7-1.1)$ & $0.9(0.7-1.2)$ & $1.1(0.9-1.4)^{a, b}$ & 0.018 \\
\hline Urate (mg/dL) & $5.2(4.5-6.2)$ & $5.4(4.2-6.3)$ & $6.4(4.8-7.4)^{\mathrm{a}, \mathrm{b}}$ & 0.033 \\
\hline Sodium $(\mathrm{mg} / \mathrm{L})$ & $140.8(139.0-141.0)$ & $140.0(138.7-141.2)$ & $140.0(138.0-142.0)$ & 0.847 \\
\hline Potassium (mg/L) & $4.5(4.2-4.7)$ & $4.5(4.3-4.9)$ & $4.7(4.1-5.0)$ & 0.350 \\
\hline Chloride (mg/L) & $104.7(103.0-106.0)$ & $106.0(102.8-107.2)$ & $104.8(103.0-107.0)$ & 0.517 \\
\hline Calcium (mg/dL) & $9.4(9.1-9.9)$ & $9.5(9.3-9.8)$ & $9.4(9.2-9.6)$ & 0.714 \\
\hline Magnesium (mg/dL) & $1.8(1.7-1.9)$ & $1.8(1.7-2.0)$ & $1.7(1.7-1.9)$ & 0.326 \\
\hline Phosphate (mg/dL) & $3.5(3.3-3.8)$ & $3.6(3.2-3.8)$ & $3.3(3.1-3.7)$ & 0.274 \\
\hline Total cholesterol (mg/dL) & $158.0(138.0-180.0)$ & $154.0(137.8-177.8)$ & $154.5(141.0-179.0)$ & 0.991 \\
\hline HDL cholesterol (mg/dL) & $42.5(36.0-48.0)$ & $39.5(31.0-46.8)$ & $38.0(32.0-53.0)$ & 0.597 \\
\hline LDL cholesterol mg/dL) & $93.0(69.2-104.8)$ & 89.8 (66.3-101.6) & $84.9(70.0-101.8)$ & 0.874 \\
\hline Triglycerides (mg/dL) & $121.0(84.0-191.0)$ & $143.5(99.8-198.5)$ & $132.0(103.0-203.0)$ & 0.354 \\
\hline Albumin $(\mathrm{g} / \mathrm{dL})$ & $4.2(4.0-4.4)$ & $4.1(4.0-4.3)$ & $4.0(3.8-4.2)$ & 0.106 \\
\hline Alkaline phosphatase $(\mathrm{u} / \mathrm{L})$ & $78.0(57.0-91.0)$ & $73.5(58.5-93.3)$ & $81.5(69.0-93.0)$ & 0.512 \\
\hline PTHi $(\mathrm{pg} / \mathrm{mL})$ & $67.0(48.9-99.6)$ & $73.0(48.9-100.3)$ & $69.7(51.8-100.3)$ & 0.993 \\
\hline 25-hydroxyvitamin D (ng/mL) & $23.0(17.0-37.2)$ & $26.8(16.0-44.0)$ & $26.8(17.9-33.3)$ & 0.670 \\
\hline $\operatorname{GFR}\left(\mathrm{mL} / \mathrm{min} / 1.73 \mathrm{~m}^{2}\right)$ & $77.8(55.7-88.9)$ & $83.1(50.1-98.2)$ & $65.4(46.0-79.1)^{\mathrm{a}, \mathrm{b}}$ & 0.040 \\
\hline Urinary creatinine $(\mathrm{mg} / \mathrm{dL})$ & $70.5(44.9-89.7)$ & $68.7(54.6-100.3)$ & $54.5(36.5-88.1)$ & 0.115 \\
\hline Urinary $\mathrm{Alb} /$ creat ratio $(\mathrm{mg} / \mathrm{g})$ & $9.5(4.2-22.2)$ & $11.2(5.5-98.6)$ & $24.5(9.4-47.5)^{\text {a }}$ & 0.017 \\
\hline Urinary albumin $(\mathrm{mg} / \mathrm{dL})$ & $0.6(0.5-3.3)$ & $0.8(0.5-12.7)$ & $1.1(0.5-7.7)$ & 0.069 \\
\hline
\end{tabular}

Each value is given as median (interquartile range). The significance of differences between groups were determined using one-way ANOVA and the independent-samples $t$-test; or Kruskal-Wallis test and Mann-Whitney U test for quantitative data. a: $p<0.05$ vs. corresponding value of Low AGEs group; b: $p<0.05$ vs. corresponding value of Intermediate AGEs group. Abbreviations. HbA1c: glycated hemoglobin; HDL: high-density lipoprotein; LDL: low-density lipoprotein; PTHi: intact parathyroid hormone; GFR: glomerular filtration rate calculated by MDRD-4 IDMS equation; Alb: albumin; Creat: creatinine.

\subsection{Abdominal Aortic Calcification and AGEs Levels}

There was a trend toward more AAC as serum AGEs levels increased (Figure 2). The median (inter-quartile range, IQR) of AAC 24-points score was 2 (0-5), 9.5 (4-13) and 8 (2-13) for low, intermediate and high AGEs groups, respectively (Figure $2 \mathrm{a}, p=0.025$ ). There were 23\%, 68\% and 63\% of patients with moderate-severe AAC in the low, intermediate and high groups respectively (Figure 2b, $p<0.001)$. Furthermore, $21(20.2 \%)$ of all patients presented no AAC and of these, $10(47.6 \%), 5(23.8 \%)$ and $6(28.6 \%)$ presented low, intermediate and high AGEs levels, respectively.

\subsection{Cardiovascular (CV) Risk Factors Associated with Abdominal Aortic Calcification (AAC)}

Table 3 shows the clinical characteristics and laboratory parameters between patients with no-mild AAC $(\mathrm{AAC}<6)$ and moderate-severe AAC $(\mathrm{AAC} \geq 6)$. The group with moderate-severe AAC was older, had a higher percentage of males, more DM2 complications, more prevalence of atherosclerosis, higher percentage of smokers or ex-smokers, lower GFR and higher levels of urinary albumin, albumin: creatinine ratio, creatinine, triglycerides, $\mathrm{HbA1c}$ and AGEs compared to those with no-mild AAC. 
Table 3. Clinical characteristics and laboratory parameters between patients with no-mild AAC and moderate-severe AAC.

\begin{tabular}{|c|c|c|c|}
\hline & $\begin{array}{l}\text { No-Mild AAC } \\
(\text { AAC }<6) \\
(n=51)\end{array}$ & $\begin{array}{c}\text { Moderate-Severe AAC } \\
(\mathrm{AAC} \geq 6) \\
(n=53)\end{array}$ & $p$-Value \\
\hline Age (years) & $67.3 \pm 7.4$ & $71.4 \pm 8.1$ & 0.011 \\
\hline Sex (female) & $33(64.7 \%)$ & $21(39.6 \%)$ & 0.012 \\
\hline Body Mass Index $\left(\mathrm{kg} / \mathrm{m}^{2}\right)$ & $31.6 \pm 4.8$ & $31.0 \pm 7.0$ & 0.174 \\
\hline Waist circumference $(\mathrm{cm})$ & $111 \pm 10$ & $108 \pm 12$ & 0.067 \\
\hline Systolic blood pressure (mmHg) & $145 \pm 17$ & $147 \pm 20$ & 0.815 \\
\hline Diastolic blood pressure ( $\mathrm{mmHg}$ ) & $77 \pm 10$ & $76 \pm 11$ & 0.951 \\
\hline Pulse pressure $(\mathrm{mmHg})$ & $69 \pm 16$ & $70 \pm 17$ & 0.682 \\
\hline Heart rate (pulse/min) & $79 \pm 12$ & $76 \pm 14$ & 0.401 \\
\hline Time from diagnosis of diabetes & & & 0.254 \\
\hline Less than 5 years & $6(11.8 \%)$ & $3(5.7 \%)$ & \\
\hline Between 5 and 10 years & $16(31.4 \%)$ & $12(22.6 \%)$ & \\
\hline More than 5 years & $29(56.9 \%)$ & $38(71.7 \%)$ & \\
\hline Diabetic complications $\left(\mathrm{n}^{\circ}\right)$ & & & 0.002 \\
\hline 1 & $10(19.6 \%)$ & $16(30.2 \%)$ & \\
\hline 2 & $9(17.6 \%)$ & $7(13.2 \%)$ & \\
\hline 3 or more & $4(7.8 \%)$ & $17(32.1 \%)$ & \\
\hline \multicolumn{4}{|c|}{ Comorbidities, toxics and clinical history } \\
\hline $\begin{array}{l}\text { Chronic kidney disease } \\
\text { (stage } 2 \text { or upper) }\end{array}$ & $34(66.7 \%)$ & $44(86.3 \%)$ & 0.071 \\
\hline Hypertension & $45(88.2 \%)$ & $48(94.1 \%)$ & 0.758 \\
\hline Smoking (current or ex) & $13(25.5 \%)$ & $27(52.9 \%)$ & 0.009 \\
\hline Alcohol (current or ex) & $8(15.7 \%)$ & $5(9.8 \%)$ & 0.386 \\
\hline Prior cardiovascular disease & $20(39.2 \%)$ & $28(54.9 \%)$ & 0.175 \\
\hline Diabetic retinopathy & $15(29.4 \%)$ & $21(39.6 \%)$ & 0.308 \\
\hline Ischemic heart disease & $5(9.8 \%)$ & $13(24.5 \%)$ & 0.069 \\
\hline Cerebral stroke & $5(9.8 \%)$ & $9(17.0 \%)$ & 0.391 \\
\hline Diabetic polyneuropathy & $9(17.6 \%)$ & $15(28.3 \%)$ & 0.247 \\
\hline Diabetic foot & $1(2.0 \%)$ & $4(7.5 \%)$ & 0.363 \\
\hline Intermittent claudication & $2(3.9 \%)$ & $8(15.1 \%)$ & 0.930 \\
\hline Atherosclerosis & $3(5.9 \%)$ & $13(24.5 \%)$ & 0.013 \\
\hline \multicolumn{4}{|c|}{ Laboratory parameters } \\
\hline AGEs $(\mathrm{U} / \mathrm{mL})$ & $5.9(4.5-10.1)$ & $9.7(7.5-11.1)$ & 0.015 \\
\hline HbA1c (\%) & $7.2(6.4-8.1)$ & $7.4(6.9-8.4)$ & 0.171 \\
\hline Leukocytes $(\times 109 / \mathrm{L})$ & $7.8(6.7-9.3)$ & $7.5(6.3-9.3)$ & 0.509 \\
\hline Hemoglobin $(\mathrm{g} / \mathrm{dL})$ & $13.4(12.5-14.5)$ & $13.0(11.6-14.5)$ & 0.164 \\
\hline Glucose $(\mathrm{mg} / \mathrm{dL})$ & $152(125-180)$ & $139(111-226)$ & 0.701 \\
\hline Creatinine $(\mathrm{mg} / \mathrm{dL})$ & $0.8(0.7-1.1)$ & $1.1(0.8-1.4)$ & 0.004 \\
\hline Urate $(\mathrm{mg} / \mathrm{dL})$ & $5.2(4.5-6.0)$ & $6.2(4.3-7.3)$ & 0.036 \\
\hline Sodium $(\mathrm{mg} / \mathrm{L})$ & $140(139-141)$ & $140(138-142)$ & 0.536 \\
\hline Potassium (mg/L) & $4.4(4.2-4.7)$ & $4.7(4.3-5.0)$ & 0.022 \\
\hline Calcium (mg/dL) & $9.5(9.2-9.8)$ & $9.4(9.2-9.7)$ & 0.577 \\
\hline Phosphate (mg/dL) & $3.5(3.2-3.8)$ & $3.4(3.1-3.8)$ & 0.320 \\
\hline Total cholesterol (mg/dL) & $158(134-180)$ & $154(141-177)$ & 0.964 \\
\hline HDL cholesterol (mg/dL) & $42.5(36.0-49.0)$ & $38.0(32.0-47.5)$ & 0.199 \\
\hline LDL cholesterol mg/dL) & $93.0(68.3-105.2)$ & $85.0(67.8-100.9)$ & 0.599 \\
\hline Triglycerides (mg/dL) & $121(94-176)$ & $149(103-203)$ & 0.048 \\
\hline Albumin (g/dL) & $4.2(4.0-4.4)$ & $4.0(3.8-4.2)$ & 0.004 \\
\hline Alkaline phosphatase $(\mathrm{u} / \mathrm{L})$ & $77(57-91)$ & $82(67-93)$ & 0.356 \\
\hline PTHi $(\mathrm{pg} / \mathrm{mL})$ & $67.0(48.9-94.6)$ & $74.2(51.8-102.9)$ & 0.333 \\
\hline 25-hydroxyvitamin D (ng/mL) & $23.0(16.3-41.0)$ & $26.8(18.0-34.0)$ & 0.547 \\
\hline GFR $(\mathrm{mL} / \mathrm{min} / \mathrm{sup})$ & $79.2(57.4-95.3)$ & $68.0(47.1-82.3)$ & 0.024 \\
\hline Urinary creatinine $(\mathrm{mg} / \mathrm{dL})$ & $70.5(52.4-89.7)$ & $60.3(39.2-89.7)$ & 0.184 \\
\hline Urinary $\mathrm{Alb} /$ creat ratio $(\mathrm{mg} / \mathrm{g})$ & $10(5-29)$ & $25(9-149)$ & 0.004 \\
\hline Urinary albumin $(\mathrm{mg} / \mathrm{dL})$ & $0.6(0.5-3.6)$ & $1.1(0.5-17.1)$ & 0.012 \\
\hline
\end{tabular}

Each value is given as mean ( \pm standard deviation), frequency (percentage) or median (interquartile range). The significance of differences between groups were determined using independent $t$-test or Mann-Whitney $\mathrm{U}$ test for quantitative data and chi-square test or Fisher's exact test for qualitative data. Abbreviations. AGEs: advanced glycation end products; HbA1c: glycated hemoglobin; HDL: high-density lipoprotein; LDL: low-density lipoprotein; PTHi: intact parathyroid hormone; GFR: glomerular filtration rate calculated by MDRD-4 IDMS equation; Alb: albumin; Creat: creatinine.

ROC curves and optimal cut off values were calculated for quantitative risk factors associated to moderate-severe AAC (Figure 3). As can be seen, circulating serum AGEs upper than $7 \mathrm{U} / \mathrm{mL}$ had a sensitivity and specificity of $81.1 \%$ and $56.9 \%$, respectively. The overall accuracy was $68.3 \%$ for AGEs above $7 \mathrm{U} / \mathrm{mL}$ and it was bigger than those for the rest of the analyzed variables $(62.5 \%$ for age above 70 years; $61.5 \%$ for triglycerides upper than $146 \mathrm{mg} / \mathrm{dL}$; and $64.4 \%$ for GFR lower than $\left.74 \mathrm{~mL} / \mathrm{min} / 1.73 \mathrm{~m}^{2}\right)$. 
(a)

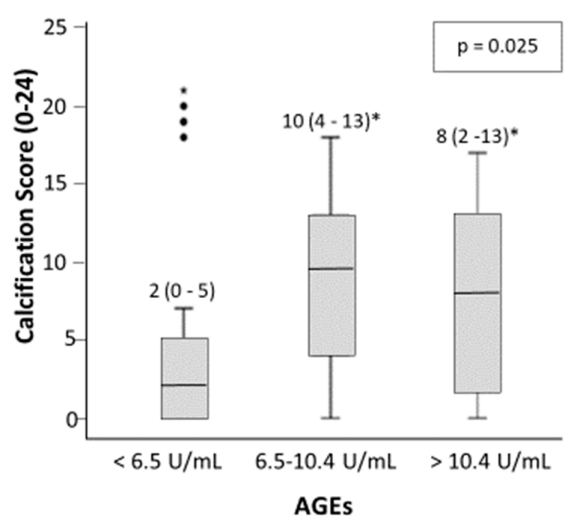

(b)

- No or mild AAC $($ AAC $<6)$

Moderate or severe AAC (AAC $\geq 6) \quad \mathrm{p}<0.001$

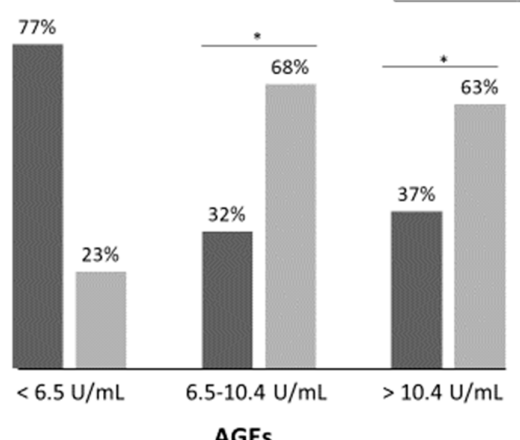

Figure 2. Abdominal aortic calcification (AAC) score (a) and percentage of patient with no-mild AAC and moderate-severe AAC (b) among AGEs levels in patients with DM2. The significance of differences between groups were determined using Kruskal-Wallis and Mann-Whitney U test for (a); and chi-square test for (b). ${ }^{*} p<0.05$ vs. low serum levels of AGEs $(<6.5 \mathrm{U} / \mathrm{mL})$.

(a)

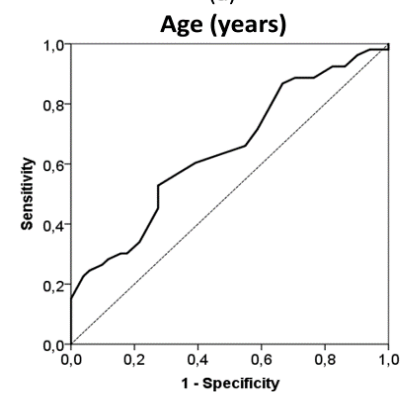

(c)
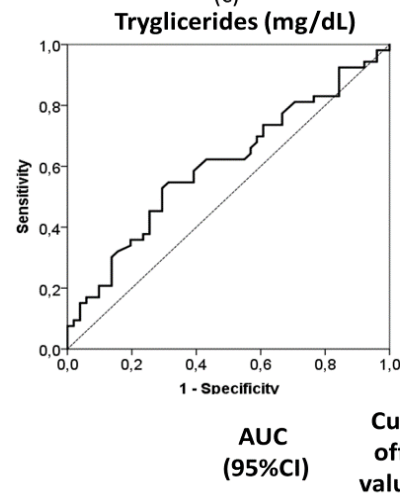

\begin{tabular}{ccc} 
& $(95 \% \mathrm{Cl})$ & valu \\
\hline Age & 0.644 & \\
(years) & $(0.539-0.750)$ & $>70$
\end{tabular}

AGEs $\quad 0.638$

$(\mathrm{U} / \mathrm{mL}) \quad(0.525-0.751)>7.0$

$\underset{(\mathrm{mg} / \mathrm{dL})}{\operatorname{Tryglicerides}}$

$(\mathrm{mg} / \mathrm{dL})$

GFR

$\begin{gathered}0.604 \\ (0.495-0.713)\end{gathered}>146$

0.628

$\left(\mathrm{mL} / \mathrm{min} / 1.73 \mathrm{~m}^{2}\right) \quad(0.520-0.736)<74$ (b)

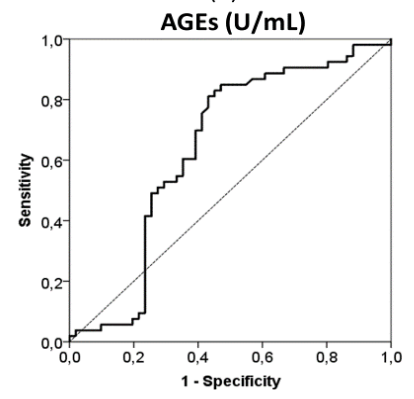

(d)

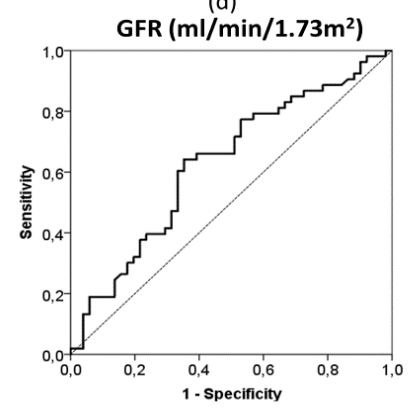

Sensitivity Specificity NPV PPV Overall

$\begin{array}{llll}\text { (\%) } & (\%) & \text { (\%) } & (\%)\end{array}$

$\begin{array}{llll}52.8 & 72.5 & 59.766 .7 & 62.5\end{array}$

$\begin{array}{llll}81.1 & 56.9 & 65.672 .5 & 68.3\end{array}$

$\begin{array}{llll}52.8 & 70.6 \quad 59.0 & 65.1 & 61.5\end{array}$

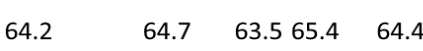

Figure 3. Receiver operating characteristic (ROC) curves and optimal cut-off of the following quantitative risk factors associated to moderate-severe AAC (vs. no-mild AAC): age (a), AGEs (b), triglycerides (c) and GFR (d). The optimal cutoff values were determined by the maximum Youden index (J), defined as sensitivity + specificity -1 . The table indicate the area under the curve (AUC), sensitivity, specificity, positive predictive value (PPV), negative predictive value (NPV) and overall accuracy of the optimal cut off values. Abbreviations. AGEs; advanced glycation end products; GFR: glomerular filtration rate. 
Univariate and multivariate logistic regression analysis were used to investigate independent factors associated to the presence of moderate-severe AAC (vs. no-mild AAC). All previously listed factors $(p<0.05$ in Table 3$)$ were included initially in the model before stepwise and backward elimination. The final model included age older than 70 years, being male, circulating AGEs levels upper than $7 \mathrm{U} / \mathrm{mL}$, and triglycerides levels upper than $146 \mathrm{mg} / \mathrm{dL}$ as the independent risk factors associated to moderate-severe AAC in DM2 (Figure 4).

(a)

Adjusted Odds Ratio

$\begin{array}{lllllllllll}0 & 1 & 2 & 3 & 4 & 5 & 6 & 7 & 8 & 9 & 1011121314\end{array}$

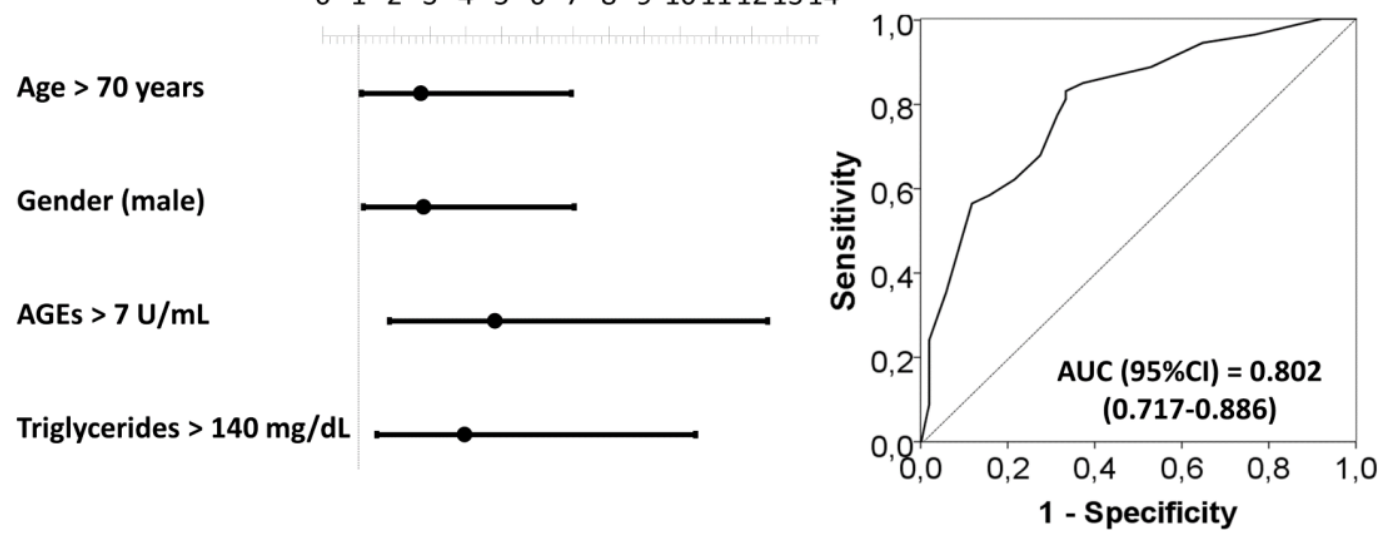

\begin{tabular}{|c|c|c|c|c|c|}
\hline & $\begin{array}{c}\text { Crude } \\
\text { O.R. }\end{array}$ & p-value & $\begin{array}{l}\text { Adj. } \\
\text { O.R. }\end{array}$ & (95\% C.I. for O.R.) & p-value \\
\hline Age (years) & $2.36(1.08-5.19)$ & 0.032 & 2.74 & $(1.08-6.96)$ & 0.033 \\
\hline Gender (male) & $2.79(1.26-6.19)$ & 0.011 & 2.82 & $(1.13-7.03)$ & 0.026 \\
\hline DM2 complications (yes vs. no) & $3.75(1.63-8.63)$ & 0.002 & & & \\
\hline Atherosclerosis (yes vs. no) & $5.20(1.38-19.54)$ & 0.015 & & & \\
\hline Smoking (current or ex vs. no) & $3.04(1.33-6.95)$ & 0.009 & & & \\
\hline AGEs $>7 \mathrm{U} / \mathrm{mL}$ & $5.71(2.01-16.22)$ & 0.001 & 4.82 & $(1.87-12.44)$ & 0.001 \\
\hline $\mathrm{GFR}<74 \mathrm{ml} / \mathrm{min} / 1.73 \mathrm{~m}^{2}$ & $2.69(1.20-6.03)$ & 0.017 & & & \\
\hline Triglycerides $>146 \mathrm{mg} / \mathrm{dL}$ & $3.28(1.47-7.32)$ & 0.004 & 3.97 & $(1.51-10.43)$ & 0.005 \\
\hline
\end{tabular}

Figure 4. Forest plot (a) and ROC curve (b) of the multivariate logistic regression of risk factors associated to moderate-severe AAC in patients with DM2. Multivariate analysis was performed using stepwise backward method. Crude and adjusted odds ratio (OR) are indicated in the table. Comparison of the expected and observed frequencies by the Hosmer-Lemeshow goodness-of-fit test $(p$-value $=0.734)$ and by ROC curve $($ AUC $=0.802 ; p<0.001)$ indicated a good fit for the model. $\mathrm{AUC}=$ the area under the curve, GFR = glomerular filtration rate.

\section{Discussion}

Our study reports that serum levels of a pool of circulating AGEs determined by ELISA positively correlated with AAC (based on lateral lumbar X-rays) in subjects with DM2. Previously, Saremi et al. prospectively demonstrated in the VA Diabetes Trial and Follow-up Study that baseline plasma levels of $\mathrm{N} \varepsilon$-carboxyethyl lysine (CEL) determined by liquid chromatography-mass spectrometry were strongly associated with the extent of AAC determined by CT scanning after an average of 10 years of follow-up [34]. However, this study did not report AGE measurements at the time of CT acquisition, so the relationship between AGE and AAC at the same time-point was not examined.

Secondly, we have also observed that both groups of patients with intermediate and high AGEs levels had a higher prevalence of diabetes-related complications. Several data have linked circulating AGEs to the development and progression of diabetes complications [35-37] including 
diabetic retinopathy [38-41], nephropathy [42,43], and cardiovascular disease [44-46]. Furthermore, an accumulation of AGEs has been detected in most of target tissues of diabetes complications, such as the kidney, retina, and atherosclerotic plaques [47]. Several studies have shown correlations between serum levels of AGEs and the development/severity of vascular disease [48-52]. Our results indicate that circulating levels of AGEs are mainly correlated with CKD and aging. These results are consistent with previous studies which found that serum AGEs are positively correlated with serum creatinine and inversely correlated with GFR [23,24,35,42,53]. Regarding aging and circulating levels of AGEs, our results are in accordance with other authors who indicated that AGEs are linked to the aging process $[35,36,47,51,54,55]$ and are considered a marker of senescence [56].

On the other hand, we find an association between circulating AGEs and HbA1c. Similar results have been found in other studies [35] where AGEs levels were higher in patients with poor glycemic control measured by HbA1c [54,57-59]. It is important to consider that the extent and duration of hyperglycemia are predicted by increased levels of $\mathrm{HbA1c}$, which is considered an acceptable marker. As mentioned above, prolonged exposure to glucose produces early AGEs and affects different proteins. An important example of early glycated proteins is $\mathrm{HbA1c}$, which is further modified through a series of reactions in $\mathrm{Hb}$-AGE. Under normal conditions, Hb-AGE makes up $0.42 \%$ of circulating hemoglobin $(\mathrm{Hb})$ levels, increasing to $0.75 \%$ in diabetic subjects [60]. Besides $\mathrm{HbA} 1 \mathrm{c}$ correlates with AGEs, AGEs-modified proteins are hardly degraded and remain in the vessels, kidney and heart for a long time, even after glycemic control has improved. Therefore, AGEs are considered to be a better index of cumulative diabetic exposure and one of the main actors of the metabolic memory observed in diabetic patients.

Concerning the factors associated to AAC, our results show that moderate-severe AAC is associated to current or ex tobacco use. Similar results were found in other studies, where cigarette smoking was strongly associated with calcification [61]. The independent factors, however, associated with AAC in our patients were age, male gender, AGEs levels and plasma triglycerides. These results are in accordance with previous studies which have reported that age and prior CV disease are strongly associated with AAC [62-64]. Triglycerides can be markers of glycemic control and have become an independent predictor of cardiovascular risk, as demonstrated by multiple prospective epidemiological studies [65-68]. Also, it is well-known that vascular calcification is more common in males than in females [69]. It is worth underlining that we developed ROC curves and searched for the optimal cut-off values associated with AAC. Remarkably, the accuracy for AGEs was higher than the observed for the other factors classically related to AAC (Figure 4). Moreover, an AUC higher than 0.8 showed a good fit for the multivariate model of factors associated to moderate-severe AAC (Figure 4). All these data suggest that AGEs could be useful markers of AAC which could identify those priority patients of diagnostic imaging techniques. The presence of calcification in any arterial wall is associated with a 3- to 4-fold higher risk for mortality and cardiovascular events [4]. Consequently, the early detection of AAC could have clinical importance in order to intensify cardiovascular prevention efforts. Therefore, it would be worth developing further prospective studies to draw definitive conclusions about the application of AGEs in algorithms for AAC prediction.

Our results support the notion that AGEs can play a role in the pathogenesis of aortic calcifications and are in accordance with tissue culture studies that indicate that AGEs can act as an inducer of calcification affecting cell function via both, intracellular glycation of proteins and AGE-RAGE signaling $[20,22,70]$.

Considering that morbidity and mortality in patients with DM2 are mainly due to cardiovascular disease, the measurement of circulating AGEs could be useful as an early marker of the presence and severity of AAC and the occurrence of future diabetic complications. In addition, an inhibitory therapy of the formation of AGEs or a therapy addressed against the AGE-RAGE system could be useful as a preventive treatment in the development of AAC and diabetic complications Recently, DNA aptamers directed against AGE and RAGE, attenuating their interaction, have been developed and are being studied with promising experimental results [71]. Also, phytate has been described 
as inhibiting glycation processes by the iron chelation process [72] and is a well-known inhibitor of AAC via absorption over the hydroxyapatite crystals. Consequently, it is possible that this compound can be a strong inhibitor of the formation of AAC via the two aforementioned mechanisms [73-75]. Interestingly, a recent study suggests that the antidiabetic therapy with a sodium-glucose cotransporter inhibitor could reduce the levels of AGEs in comparison with a dipeptidyl peptidase-4 inhibitor [76]. Finally, beside drugs or compounds, the avoidance of smoking and the restriction of dietary AGEs consumption must be borne in mind to reduce the AGEs burden $[77,78]$.

Our study has several limitations. First, the sample is small and it is a cross-sectional study from a single medical center; therefore, the findings presented here should be interpreted with caution. Even though we found that age, gender, triglycerides and circulating levels of AGEs were associated to AAC, this does not prove causality. Prospective multicentric longitudinal studies are needed to fully clarify how AGEs actually contribute to the genesis and progression of AAC and to assess the possibility that circulating levels of AGEs may serve as biomarkers to predict or diagnose AAC. Another possible limitation is that we measured AAC using lateral abdominal plain X-rays. One could argue that $\mathrm{CT}$ is more sensitive. Several studies, however, have positioned lateral lumbar radiography as a tool to estimate the severity of AAC. Furthermore, AAC evaluated by lateral X-rays shows a good correlation with coronary calcium score $[4,79]$. Therefore, we consider that plain radiography is an appropriate screening method for evaluating AAC in daily practice since it is simpler, cheaper and with lesser radiation than $\mathrm{CT}$.

Finally, there are several methods available to measure AGEs and there are no single standardized protocols or normal reference ranges established for them. We measured a pool of AGEs by ELISA. It has been observed that CEL but not methylglyoxal hydroimidazolone, 3-deoxyglucosone hydroimidazolone, 2-aminoadipic acid and methionine sulfoxide was related to AAC [34]. Therefore, it is possible that our results were related to specific AGEs. Thus, further studies aimed at addressing these issues are needed.

\section{Conclusions}

In conclusion, we provide evidence that serum levels of AGEs positively correlate with AAC in patients with DM2. In addition, our study suggests that age, gender, triglycerides and circulating levels of AGEs are the risk factors associated to moderate-severe AAC. We also have shown that circulating levels of AGEs were positively associated with AAC severity and diabetes-related complications. Therefore, increased levels of AGEs can be contemplated as a biomarker of AAC and other vascular complications of diabetes.

Author Contributions: All authors make substantial contributions to conception and design, and/or acquisition of data, and/or analysis and interpretation of data: conceptualization, P.S., L.M. and F.G.; methodology, P.S., R.R., M.M.-G. and L.M.; software, M.M.-G., P.S. and R.R.; validation, P.S., R.R. and L.M.; formal analysis, P.S and R.R.; investigation and resources, R.R., P.S., R.F., M.G.-F., M.M. and C.R.; data curation, R.R., and P.S.; writing-original draft preparation, P.S., R.R. and L.M.; writing-review and editing L.M., F.G., R.R., M.G.-F. and P.S.; visualization, L.M., R.R. and P.S.; supervision, L.M., F.G. and P.S.; project administration L.M., F.G. and P.S.; funding acquisition, L.M., F.G. and P.S. All authors have read and agreed to the published version of the manuscript.

Funding: This research was funded by Balearic Islands Government, grant number AAEE033/2017.

Acknowledgments: MGF acknowledges funding by the Miguel Servet Program (MS19/00201), Instituto de Salud Carlos III (ISCIII), Madrid. All authors acknowledge funding by the LIBERI program, Health Research Institute of the Balearic Islands (IdISBa), Palma de Mallorca.

Conflicts of Interest: The authors declare no conflict of interest.

\section{References}

1. Cosentino, F.; Grant, P.J.; Aboyans, V.; Bailey, C.J.; Ceriello, A.; Delgado, V.; Federici, M.; Filippatos, G.; Grobbee, D.E.; Hansen, T.B.; et al. 2019 ESC Guidelines on diabetes, pre-diabetes, and cardiovascular diseases developed in collaboration with the EASD. Eur. Hear. J. 2019, 41, 255-323. [CrossRef] [PubMed] 
2. Bendix, E.F.; Johansen, E.; Ringgaard, T.; Wolder, M.; Starup-Linde, J. Diabetes and Abdominal Aortic Calcification-A Systematic Review. Curr. Osteoporos. Rep. 2018, 16, 42-57. [CrossRef] [PubMed]

3. Kalofoutis, C.; Piperi, C.; Kalofoutis, A.; Harris, F.; Phoenix, D.; Singh, J. Type II diabetes mellitus and cardiovascular risk factors: Current therapeutic approaches. Exp. Clin. Cardiol. 2007, 12, 17-28. [PubMed]

4. Gonçalves, F.B.; Voûte, M.; Hoeks, S.E.; Chonchol, M.B.; Boersma, E.E.; Stolker, R.J.; Verhagen, H.J.M. Calcification of the abdominal aorta as an independent predictor of cardiovascular events: A meta-analysis. Heart 2012, 98, 988-994. [CrossRef] [PubMed]

5. Cho, A.; Jung, H.Y.; Park, H.C.; Oh, J.; Kim, J.; Lee, Y.-K. Relationship between abdominal aortic calcification on plain radiograph and coronary artery calcification detected by computed tomography in hemodialysis patients. Clin. Nephrol. 2020, 93, 123-129. [CrossRef] [PubMed]

6. Zhu, X.; Cai, H.; Zhu, M.; Zhan, Y.; Che, M.; Lin, X.; Zhang, W.; Ni, Z. Association of abdominal aortic calcification estimated by plain radiography with outcomes in haemodialysis patients: A 6-year follow-up study. Nephrology 2019. [CrossRef]

7. Oishi, H.; Horibe, H.; Yamase, Y.; Ueyama, C.; Takemoto, Y.; Shigeta, T.; Hibino, T.; Kondo, T.; Suzuki, S.; Ishii, H.; et al. Predictive value of abdominal aortic calcification index for mid-term cardiovascular events in patients with acute coronary syndrome. Hear. Vessel. 2019, 35, 620-629. [CrossRef]

8. Schousboe, J.T.; Vo, T.N.; Langsetmo, L.; Adabag, S.; Szulc, P.; Lewis, J.R.; Kats, A.M.; Taylor, B.C.; Ensrud, K.E. Abdominal aortic calcification (AAC) and ankle-brachial index (ABI) predict health care costs and utilization in older men, independent of prevalent clinical cardiovascular disease and each other. Atherosclerosis 2020, 295, 31-37. [CrossRef]

9. Giachelli, C.M. Vascular Calcification Mechanisms. J. Am. Soc. Nephrol. 2004, 15, 2959-2964. [CrossRef]

10. Wu, M.; Rementer, C.; Giachelli, C.M. Vascular calcification: An update on mechanisms and challenges in treatment. Calcif. Tissue Int. 2013, 93, 365-373. [CrossRef]

11. Demer, L.L.; Tintut, Y. Vascular Calcification. Circulation 2008, 117, 2938-2948. [CrossRef] [PubMed]

12. Pescatore, L.A.; Gamarra, L.F.; Liberman, M. Multifaceted Mechanisms of Vascular Calcification in Aging. Arter. Thromb. Vasc. Boil. 2019, 39, 1307-1316. [CrossRef] [PubMed]

13. Harper, E.; Forde, H.; Davenport, C.; Rochfort, K.; Smith, D.; Cummins, P.M. Vascular calcification in type-2 diabetes and cardiovascular disease: Integrative roles for OPG, RANKL and TRAIL. Vasc. Pharmacol. 2016, 82, 30-40. [CrossRef] [PubMed]

14. Schlieper, G.; Schurgers, L.; Brandenburg, V.; Reutelingsperger, C.; Floege, J. Vascular calcification in chronic kidney disease: An update. Nephrol. Dial. Transplant. 2015, 31, 31-39. [CrossRef] [PubMed]

15. Chen, N.X.; Moe, S.M. Pathophysiology of Vascular Calcification. Curr. Osteoporos. Rep. 2015, 13, 372-380. [CrossRef] [PubMed]

16. Setiawati, R.; di Chio, F.; Rahardjo, P.; Nasuto, M.; Dimpudus, F.J.; Guglielmi, G.; Information, P.E.K.F.C. Quantitative Assessment of Abdominal Aortic Calcifications Using Lateral Lumbar Radiograph, Dual-Energy X-ray Absorptiometry, and Quantitative Computed Tomography of the Spine. J. Clin. Densitom. 2016, 19, 242-249. [CrossRef]

17. Negre-Salvayre, A.; Salvayre, R.; Augé, N.; Pamplona, R.; Portero-Otín, M. Hyperglycemia and Glycation in Diabetic Complications. Antioxid. Redox Signal. 2009, 11, 3071-3109. [CrossRef]

18. Peppa, M.; Vlassara, H. Advanced glycation end products and diabetic complications: A general overview. Hormones 2006, 4, 28-37. [CrossRef]

19. Brownlee, M.M. Advanced protein glycosylation in diabetes and aging. Annu. Rev. Med. 1995, 46, $223-234$. [CrossRef]

20. Aronson, D. Cross-linking of glycated collagen in the pathogenesis of arterial and myocardial stiffening of aging and diabetes. J. Hypertens. 2003, 21, 3-12. [CrossRef]

21. Bierhaus, A.; Humpert, P.M.; Stern, D.M.; Arnold, B.; Nawroth, P.P. Advanced Glycation End Product Receptor?Mediated Cellular Dysfunction. Ann. N. Y. Acad. Sci. 2005, 1043, 676-680. [CrossRef] [PubMed]

22. Kay, A.M.; Simpson, C.L.; Stewart, J.A. The Role of AGE/RAGE Signaling in Diabetes-Mediated Vascular Calcification. J. Diabetes Res. 2016, 2016, 6809703. [CrossRef] [PubMed]

23. Taki, K.; Takayama, F.; Tsuruta, Y.; Niwa, T. Oxidative stress, advanced glycation product, and coronary artery calcification in hemodialysis patients. Kidney Int. 2006, 70, 218-224. [CrossRef] 
24. Nasrallah, M.M.; El-Shehaby, A.R.; Osman, N.A.; Salem, M.M.; Nassef, A.; El Din, U.A.A.S. Erratum to: Endogenous soluble receptor of advanced glycation end-products (esRAGE) is negatively associated with vascular calcification in non-diabetic hemodialysis patients. Int. Urol. Nephrol. 2011, 44, 1201-1202. [CrossRef]

25. Ren, X.; Shao, H.; Wei, Q.; Sun, Z.; Liu, N. Advanced Glycation End-products Enhance Calcification in Vascular Smooth Muscle Cells. J. Int. Med Res. 2009, 37, 847-854. [CrossRef] [PubMed]

26. Tanikawa, T.; Okada, Y.; Tanikawa, R.; Tanaka, Y. Advanced Glycation End Products Induce Calcification of Vascular Smooth Muscle Cells through RAGE/p38 MAPK. J. Vasc. Res. 2009, 46, 572-580. [CrossRef] [PubMed]

27. Suga, T.; Iso, T.; Shimizu, T.; Tanaka, T.; Yamagishi, S.-I.; Takeuchi, M.; Imaizumi, T.; Kurabayashi, M. Activation of receptor for advanced glycation end products induces osteogenic differentiation of vascular smooth muscle cells. J. Atheroscler. Thromb. 2011, 18, 670-683. [CrossRef]

28. Gawdzik, J.; Mathew, L.; Kim, G.; Puri, T.S.; Bowman, M.H. Vascular remodeling and arterial calcification are directly mediated by S100A12 (EN-RAGE) in chronic kidney disease. Am. J. Nephrol. 2011, 33, $250-259$. [CrossRef]

29. Zhu, Y.; Ma, W.-Q.; Han, X.-Q.; Wang, Y.; Wang, X.; Liu, N.-F. Advanced glycation end products accelerate calcification in VSMCs through HIF-1 $\alpha /$ PDK4 activation and suppress glucose metabolism. Sci. Rep. 2018, 8, 13730. [CrossRef]

30. Chobanian, A.V.; Bakris, G.L.; Black, H.R.; Cushman, W.C.; Green, L.A.; Izzo, J.L., Jr.; Jones, D.W.; Materson, B.J.; Oparil, S.; Wright, J.T., Jr.; et al. National Heart, Lung, and Blood Institute; National High Blood Pressure Education Program Coordinating Committee. Seventh report of the Joint National Committee on Prevention, Detection, Evaluation, and Treatment of High Blood Pressure. Hypertension 2003, 42, 1206-1252. [CrossRef]

31. National Kidney Foundation. K/DOQI clinical practice guidelines for chronic kidney disease: Evaluation, classification, and stratification. Am. J. Kidney Dis. 2002, 39, S1-S266. [CrossRef]

32. Honkanen, E.; Kauppila, L.; Wikström, B.; Rensma, P.L.; Krzesinski, J.-M.; Aasarod, K.; Verbeke, F.; Jensen, P.B.; Mattelaer, P.; Volck, B.; et al. Abdominal aortic calcification in dialysis patients: Results of the CORD study. Nephrol. Dial. Transplant. 2008, 23, 4009-4015. [CrossRef] [PubMed]

33. Schousboe, J.T.; Wilson, K.E.; Hangartner, T.N. Detection of Aortic Calcification during Vertebral Fracture Assessment (VFA) Compared to Digital Radiography. PLoS ONE 2007, 2, e715. [CrossRef] [PubMed]

34. Saremi, A.; Howell, S.; Schwenke, D.C.; Bahn, G.; Beisswenger, P.J.; Reaven, P.D. Advanced Glycation End Products, Oxidation Products, and the Extent of Atherosclerosis During the VA Diabetes Trial and Follow-up Study. Diabetes Care 2017, 40, 591-598. [CrossRef]

35. Thomas, M.C.; Woodward, M.; Neal, B.; Li, Q.; Pickering, R.; Marre, M.; Williams, B.; Perkovic, V.; Cooper, M.E.; Zoungas, S.; et al. Relationship Between Levels of Advanced Glycation End Products and Their Soluble Receptor and Adverse Outcomes in Adults With Type 2 Diabetes. Diabetes Care 2015, 38, 1891-1897. [CrossRef]

36. Fishman, S.L.; Sonmez, H.; Basman, C.; Singh, V.; Poretsky, L. The role of advanced glycation end-products in the development of coronary artery disease in patients with and without diabetes mellitus: A review. Mol. Med. 2018, 24, 59. [CrossRef] [PubMed]

37. Jiménez, I.U.; Díaz-Díaz, E.; Castro, J.S.; Ramos, J.P.; León, M.C.; Ríos, J.A.A.; Bautista, J.C.A.; Correa-Rotter, R.; Salinas, C.A.A.; Larrea, F. Circulating Concentrations of Advanced Glycation end Products, its Association With the Development of Diabetes Mellitus. Arch. Med Res. 2017, 48, 360-369. [CrossRef] [PubMed]

38. Wautier, M.P.; Massin, P.; Guillausseau, P.J.; Huijberts, M.; Levy, B.; Boulanger, E.; Laloi-Michelin, M.; Wautier, M. N(carboxymethyl)lysine as a biomarker for microvascular complications in type 2 diabetic patients. Diabetes Metab. 2003, 29, 44-52. [CrossRef]

39. Boehm, B.O.; Schilling, S.; Rösinger, S.; Lang, G.; Lang, G.; Kientsch-Engel, R.; Stahl, P. Elevated serum levels of N?-carboxymethyl-lysine, an advanced glycation end product, are associated with proliferative diabetic retinopathy and macular oedema. Diabetology 2004, 47, 1376-1379. [CrossRef] [PubMed]

40. Fosmark, D.S.; Torjesen, P.A.; Kilhovd, B.K.; Berg, T.J.; Sandvik, L.; Hanssen, K.F.; Agardh, C.-D.; Agardh, E. Increased serum levels of the specific advanced glycation end product methylglyoxal-derived hydroimidazolone are associated with retinopathy in patients with type 2 diabetes mellitus. Metaolism 2006, 55, 232-236. [CrossRef] [PubMed] 
41. Xu, J.; Chen, L.-J.; Yu, J.; Wang, H.-J.; Zhang, F.; Liu, Q.; Wu, J. Involvement of Advanced Glycation End Products in the Pathogenesis of Diabetic Retinopathy. Cell. Physiol. Biochem. 2018, 48, 705-717. [CrossRef]

42. Shimoike, T.; Inoguchi, T.; Umeda, F.; Nawata, H.; Kawano, K.; Ochi, H. The meaning of serum levels of advanced glycosylation end products in diabetic nephropathy. Metabolism 2000, 49, 1030-1035. [CrossRef] [PubMed]

43. Prevost, G.; Fajardy, I.; Besmond, C.; Balkau, B.; Tichet, J.; Fontaine, P.; Danze, P.M.; Marre, M.; DESIR Studies. Polymorphisms of the receptor of advanced glycation endproducts (RAGE) and the development of nephropathy in type 1 diabetic patients. Diabetes Metab. 2005, 31, 35-39. [CrossRef]

44. Bucala, R.; Makita, Z.; Vega, G.; Grundy, S.; Koschinsky, T.; Cerami, A.; Vlassara, H. Modification of low-density lipoprotein by advanced glycation end products contributes to the dyslipidemia of diabetes and renal insufficiency. Proc. Natl. Acad. Sci. USA 1994, 91, 9441-9445. [CrossRef] [PubMed]

45. Meerwaldt, R.; Links, T.P.; Zeebregts, C.; Tio, R.; Hillebrands, J.-L.; Smit, A.J. The clinical relevance of assessing advanced glycation endproducts accumulation in diabetes. Cardiovasc. Diabetol. 2008, 7, 29. [CrossRef] [PubMed]

46. Gawandi, S.; Gangawane, S.; Chakrabarti, A.; Kedare, S.; Bantwal, K.; Wadhe, V.; Kulkarni, A.; Kulkarni, S.; Rajan, M.G.R. A Study of Microalbuminuria (MAU) and Advanced Glycation End Products (AGEs) Levels in Diabetic and Hypertensive Subjects. Indian J. Clin. Biochem. 2017, 33, 81-85. [CrossRef]

47. Singh, R.; Barden, A.; Mori, T.A.; Beilin, L. Advanced glycation end-products: A review. Diabetology 2001, 44, 129-146. [CrossRef]

48. Brownlee, M. The pathological implications of protein glycation. Clin. Investig. Med. 1995, 18, $275-281$.

49. Sell, D.R.; Monnier, V.M. Molecular Basis of Arterial Stiffening: Role of Glycation? A Mini-Review. Gerontology 2012, 58, 227-237. [CrossRef]

50. Kilhovd, B.K.; Berg, T.J.; Birkeland, K.I.; Thorsby, P.M.; Hanssen, K.F. Serum levels of advanced glycation end products are increased in patients with type 2 diabetes and coronary heart disease. Diabetes Care 1999, 22, 1543-1548. [CrossRef]

51. Koska, J.; Saremi, A.; Howell, S.; Bahn, G.; De Courten, B.; Ginsberg, H.; Beisswenger, P.J.; Reaven, P.D.; For the VADT Investigators. Advanced Glycation End Products, Oxidation Products, and Incident Cardiovascular Events in Patients with Type 2 Diabetes. Diabetes Care 2017, 41, 570-576. [CrossRef] [PubMed]

52. Yozgatli, K.; Brouwer, T.; Jager, J.; Cabezas, M.C.; Lefrandt, J.D.; Noordzij, M.J.; Oomen, P.H.N.; Smit, A.J. Accumulation of advanced glycation end products is associated with macrovascular events and glycaemic control with microvascular complications in Type 2 diabetes mellitus. Diabet. Med. 2018, 35, 1242-1248. [CrossRef] [PubMed]

53. Nasrallah, M.M.; El-Shehaby, A.R.; Osman, N.A.; Salem, M.M.; Nassef, A.; El Din, U.A.A.S. Endogenous soluble receptor of advanced glycation end-products (esRAGE) is negatively associated with vascular calcification in non-diabetic hemodialysis patients. Int. Urol. Nephrol. 2011, 44, 1193-1199. [CrossRef] [PubMed]

54. Guerin-Dubourg, A.; Cournot, M.; Planesse, C.; Debussche, X.; Meilhac, O.; Rondeau, P.; Bourdon, E. Association between Fluorescent Advanced Glycation End-Products and Vascular Complications in Type 2 Diabetic Patients. BioMed Res. Int. 2017, 2017, 7989180. [CrossRef]

55. Hangai, M.; Takebe, N.; Honma, H.; Sasaki, A.; Chida, A.; Nakano, R.; Togashi, H.; Nakagawa, R.; Oda, T.; Matsui, M.; et al. Association of Advanced Glycation End Products with coronary Artery Calcification in Japanese Subjects with Type 2 Diabetes as Assessed by Skin Autofluorescence. J. Atheroscler. Thromb. 2016, 23, 1178-1187. [CrossRef] [PubMed]

56. Simm, A.; Wagner, J.; Gursinsky, T.; Nass, N.; Friedrich, I.; Schinzel, R.; Czeslik, E.; Silber, R.; Scheubel, R. Advanced glycation endproducts: A biomarker for age as an outcome predictor after cardiac surgery? Exp. Gerontol. 2007, 42, 668-675. [CrossRef]

57. Okura, T.; Ueta, E.; Nakamura, R.; Fujioka, Y.; Sumi, K.; Matsumoto, K.; Shoji, K.; Matsuzawa, K.; Izawa, S.; Nomi, Y.; et al. High Serum Advanced Glycation End Products Are Associated with Decreased Insulin Secretion in Patients with Type 2 Diabetes: A Brief Report. J. Diabetes Res. 2017, 2017, 5139750. [CrossRef] [PubMed] 
58. Assiri, A.M.A.; Kamel, H.F.; Alrefai, A. Critical Appraisal of Advanced Glycation End Products (AGEs) and Circulating Soluble Receptors for Advanced Glycation End Products (sRAGE) as a Predictive Biomarkers for Cardiovascular Disease in Hemodialysis Patients. Med. Sci. 2018, 6, 38. [CrossRef] [PubMed]

59. van Eupen, M.G.; Schram, M.T.; Colhoun, H.M.; Scheijen, J.L.; Stehouwer, C.D.; Schalkwijk, C.G. Plasma levels of advanced glycation end products are associated with type 1 diabetes and coronary artery calcification. Cardiovasc. Diabetol. 2013, 12, 149. [CrossRef]

60. Saleh, J. Glycated hemoglobin, and its spinoffs: Cardiovascular disease markers or risk factors? World J. Cardiol. 2015, 7, 449-453. [CrossRef]

61. Jung, J.-G.; Wu, L.-T.; Kim, J.S.; Kim, E.-D.; Yoon, S.J. Relationship between Smoking and Abdominal Aorta Calcification on Computed Tomography. Korean J. Fam. Med. 2019, 40, 248-253. [CrossRef] [PubMed]

62. Kauppila, L.; Polak, J.F.; Cupples, L.A.; Hannan, M.T.; Kiel, D.P.; Wilson, P.W. New indices to classify location; severity and progression of calcific lesions inthe abdominal aorta: A 25-year follow-up study. Atherosclerosis 1997, 132, 245-250. [CrossRef] [PubMed]

63. Allison, M.; Criqui, M.H.; Wright, C.M. Patterns and Risk Factors for Systemic Calcified Atherosclerosis. Arter. Thromb. Vasc. Boil. 2004, 24, 331-336. [CrossRef] [PubMed]

64. Nguyen, P.T.; Coche, E.; Goffin, E.; Beguin, C.; Vlassenbroek, A.; Devuyst, O.; Robert, A.; Jadoul, M. Prevalence and Determinants of Coronary and Aortic Calcifications Assessed by Chest CT in Renal Transplant Recipients. Am. J. Nephrol. 2007, 27, 329-335. [CrossRef]

65. Quispe, R.; Martin, S.S.; Jones, S.R. Triglycerides to high-density lipoprotein-cholesterol ratio, glycemic control, and cardiovascular risk in obese patients with type 2 diabetes. Curr. Opin. Endocrinol. Diabetes Obes. 2016, 23, 150-156. [CrossRef]

66. Egeland, G.M.; Igland, J.; Sulo, G.; Nygård, O.; Ebbing, M.; Tell, G.S. Non-fasting triglycerides predict incident acute myocardial infarction among those with favourable HDL-cholesterol: Cohort Norway. Eur. J. Prev. Cardiol. 2014, 22, 872-881. [CrossRef]

67. Sarwar, N.; Danesh, J.; Eiriksdottir, G.; Sigurdsson, G.; Wareham, N.; Bingham, S.; Boekholdt, S.M.; Khaw, K.-T.; Gudnason, V. Triglycerides and the Risk of Coronary Heart Disease. Circulation 2007, 115, 450-458. [CrossRef]

68. Nichols, G.A.; Philip, S.; Reynolds, K.; Granowitz, C.B.; Fazio, S. Increased Cardiovascular Risk in Hypertriglyceridemic Patients with Statin-Controlled LDL Cholesterol. J. Clin. Endocrinol. Metab. 2018, 103, 3019-3027. [CrossRef]

69. Leopold, J.A. Vascular calcification: Mechanisms of vascular smooth muscle cell calcification. Trends Cardiovasc. Med. 2014, 25, 267-274. [CrossRef]

70. Brownlee, M. Biochemistry, and molecular cell biology of diabetic complications. Nature 2001, 414, 813-820. [CrossRef]

71. Yamagishi, S.-I.; Matsui, T.; Yamagish, S.-I. Therapeutic Potential of DNA-aptamers Raised Against AGE-RAGE Axis in Diabetes-related Complications. Curr. Pharm. Des. 2018, 24, 2802-2809. [CrossRef] [PubMed]

72. Sanchis, P.; Rivera, R.; Berga, F.; Fortuny, R.; Adrover, M.; Costa-Bauzà, A.; Grases, F.; Masmiquel, L. Phytate Decreases Formation of Advanced Glycation End-Products in Patients with Type II Diabetes: Randomized Crossover Trial. Sci. Rep. 2018, 8, 9619. [CrossRef] [PubMed]

73. Fernandez-Palomeque, C.; Grau, A.; Perelló, J.; Sanchis, P.; Isern, B.; Prieto, R.M.; Costa-Bauzá, A.; Caldés, O.J.; Bonnin, O.; García-Raja, A.; et al. Relationship between Urinary Level of Phytate and Valvular Calcification in an Elderly Population: A Cross-Sectional Study. PLoS ONE 2015, 10, e0136560. [CrossRef] [PubMed]

74. Sanchis, P.; Buades, J.M.; Berga, F.; Gelabert, M.M.; Molina, M.; Íñigo, M.V.; García, S.; Gonzalez, J.; Bernabeu, M.R.; Costa-Bauzá, A.; et al. Protective Effect of Myo-Inositol Hexaphosphate (Phytate) on Abdominal Aortic Calcification in Patients With Chronic Kidney Disease. J. Ren. Nutr. 2016, 26, 226-236. [CrossRef] [PubMed]

75. Grases, F. Phytate reduces age-related cardiovascular calcification. Front. Biosci. 2008, 13, 7115. [CrossRef] [PubMed]

76. Bekki, M.; Tahara, N.; Tahara, A.; Igata, S.; Honda, A.; Sugiyama, Y.; Nakamura, T.; Sun, J.; Kumashiro, Y.; Matsui, T.; et al. Switching Dipeptidyl Peptidase-4 Inhibitors to Tofogliflozin, a Selective Inhibitor of Sodium-Glucose Cotransporter 2 Improve Arterial Stiffness Evaluated by Cardio-Ankle Vascular Index in Patients with Type 2 Diabetes: A Pilot Study. Curr. Vasc. Pharmacol. 2019, 17, 411-420. [CrossRef] 
77. Vlassara, H. Advanced Glycation in Health and Disease: Role of the Modern Environment. Ann. N. Y. Acad. Sci. 2005, 1043, 452-460. [CrossRef]

78. Yamagishi, S.-I.; Matsui, T. Pathologic role of dietary advanced glycation end products in cardiometabolic disorders, and therapeutic intervention. J. Nutr. 2016, 32, 157-165. [CrossRef]

79. Wilson, P.W.F.; Kauppila, L.I.; O’Donnell, C.J.; Kiel, D.P.; Hannan, M.; Polak, J.M.; Cupples, A. Abdominal Aortic Calcific Deposits Are an Important Predictor of Vascular Morbidity and Mortality. Circulation 2001, 103, 1529-1534. [CrossRef]

(C) 2020 by the authors. Licensee MDPI, Basel, Switzerland. This article is an open access article distributed under the terms and conditions of the Creative Commons Attribution (CC BY) license (http://creativecommons.org/licenses/by/4.0/). 